\title{
Semantic facilitation in bilingual everyday speech comprehension
}

\author{
Marco van de Ven ${ }^{1}$, Benjamin V. Tucker ${ }^{2}$, Mirjam Ernestus ${ }^{3,1}$ \\ ${ }^{1}$ Max Planck Institute for Psycholinguistics, The Netherlands \\ ${ }^{2}$ University of Alberta, Canada \\ ${ }^{3}$ Radboud University Nijmegen, The Netherlands \\ Marco.vandeVen@mpi.nl, bvtucker@ualberta.ca, Mirjam.Ernestus@mpi.nl
}

\begin{abstract}
Previous research suggests that bilinguals presented with low and high predictability sentences benefit from semantics in clear but not in conversational speech [1]. In everyday speech, however, many words are not highly predictable. Previous research has shown that native listeners can use also more subtle semantic contextual information [2]. The present study reports two auditory lexical decision experiments investigating to what extent late Asian-English bilinguals benefit from subtle semantic cues in their processing of English unreduced and reduced speech. Our results indicate that these bilinguals are less sensitive to semantic cues than native listeners for both speech registers.

Index Terms: acoustic reduction, word recognition, speech perception, semantics, latent semantic analysis, English, Asian, bilinguals
\end{abstract}

\section{Introduction}

In casual speech, words are often much shorter and have fewer segments than in clear speech [3, 4]. For example, the English word apparently and the Dutch word eigenlijk 'actually' can be pronounced like [' $\mathrm{p}^{\mathrm{h}}$ erĩ] and ['cik], respectively. These types of acoustically reduced pronunciation variants are highly frequent in casual speech. To illustrate, in an earlier version of the Buckeye Corpus of American English conversational speech (88,000 word tokens), complete syllables are absent in six percent of the words [3], and in the Ernestus corpus of casual Dutch (91,718 word tokens) [4], ca. $19.4 \%$ of the syllables are missing [5].

Native listeners have difficulty understanding these highly reduced pronunciation variants in isolation [6]. Previous research indicates that they can use both acoustic and semantic/syntactic cues in the context to deduce reduced words, but they favour acoustic cues whenever these two are in conflict [7]. Further, they benefit more from semantic contextual information when listening to unreduced speech compared to reduced speech [2].

Whereas several studies have thus shed some light on how native listeners deal with reduced pronunciations, it is unclear how non-native listeners do so. The present study investigates whether late Asian-English bilinguals (native speakers of an Asian language who acquired English after childhood) can make use of subtle semantic cues in their processing of unreduced and reduced pronunciation variants. We selected AsianEnglish bilinguals because Asian languages and the English language are typologically highly distinct and share few cognates, which implies that semantic priming only occurs if the speakers know the English words (instead of just the cognates in their native language).

Previous psycholinguistic research suggests that the role of semantic contextual information is highly similar in the processing of bilinguals' native (L1) and non-native (L2) languages [8].

However, other studies have shown that there are also crucial differences between native and bilingual listeners in their use of semantic contextual information. For example, when listening to speech in noise, non-native listeners benefit less from semantic/syntactic contextual information [9]. Similarly, bilinguals only benefit from semantic cues in the context when presented with clear speech (rather than conversational speech) [1].

Importantly, these studies are based on sentences, which means that the role of semantic contextual information cannot be separated from other types of higher level information (e.g., syntax and pragmatics). Further, these studies tested bilinguals' processing of high predictability (e.g., A bicycle has two wheels.) versus low predictability (e.g., Dad looked at the pork.) sentences. However, in everyday speech comprehension, many words are not highly predictable, and listeners often have to resort to much more subtle semantic contextual information.

As mentioned above, previous research has shown that native listeners use subtle semantic contextual information if they are presented with unreduced pronunciation variants, whereas they use this information to a smaller extent if presented with reduced variants [2]. The present study investigates whether bilinguals are as sensitive to subtle semantic information as native listeners in their processing of unreduced and reduced pronunciation variants. On the basis of the findings by $[1,2,9]$, we predict that monolingual and bilingual listeners show similar sensitivity to semantic cues in the processing of unreduced speech, but no sensitivity to semantic cues in the processing of reduced speech.

We report two auditory lexical decision experiments with semantic priming, in which bilinguals were presented with unreduced (Experiment 1) or reduced (Experiment 2) pronunciation variants. Similar to [2], listeners were presented with isolated words, rather than with sentences, in order to isolate the effects of semantic facilitation from the influences of other sources of higher level information.

Since this study focuses on the role of subtle semantic cues, we needed a fine-grained measure of words' semantic relatedness. For this purpose we used Latent Semantic Analysis (LSA) [10], which is a computational technique that estimates words' semantic relatedness on the basis of the numbers of direct and indirect co-occurrences of words in paragraphs or texts, extracted from a large collection of written corpora. LSA scores range from -1 to 1 , where values closer to -1 indicate a low and values closer to 1 indicate a high semantic relatedness between words. Previous research has shown that LSA scores predict semantic priming effects obtained in auditory lexical decision tasks for native listeners $[2,11,12]$. 


\section{Experiment 1}

\subsection{Introduction}

We investigated whether a word's semantic relatedness to its preceding stimulus influences lexical decision times differently for native listeners (i.e. the control group) than for bilinguals, by replicating Experiment 1 of [2] with bilingual speakers. This experiment contains word pairs with LSA scores ranging from 0.36 to 0.93 , which includes word pairs with members that are mildly, moderately, and highly related. The members of each word pair were presented in consecutive trials.

\subsection{Participants}

Twenty undergraduate students from the University of Alberta, Department of Linguistics participant pool took part in the experiment, and received course credit for their participation. They were all late Asian-English bilinguals (i.e. they acquired English after childhood) whose native languages were Mandarin $(n=11)$, Cantonese $(n=7)$, Korean $(n=1)$, and Japanese $(\mathrm{n}=1)$. They had passed a TOEFL (Test of English as a Foreign Language) examination with a minimum score of 580 (or equivalent). The data from these twenty bilinguals are compared to the data from the twenty native speakers of Canadian English who were tested in Experiment 1 in [2].

\subsection{Materials}

The stimuli are those of Experiment 1 in [2]. The 154 target nouns have widely varying word frequencies (range: 4058322), which were estimated on the basis of frequency counts for the spoken section of the Corpus of Contemporary American English (385 million word tokens) [13]. These target nouns form 77 word pairs with LSA scores ranging from mildly related (e.g., snake - beak, LSA score: 0.36) to highly related (e.g., gold - silver, LSA score: 0.88 ) word pairs. Thus, if any effects of semantic similarity show up for the target stimuli in our experiment, these effects are the result of listeners' sensitivity to subtle distinctions in semantic relatedness. In addition, the experiment contained 87 semantically unrelated filler pairs and 128 pseudowords. The limited number of pseudowords induces a "YES"-response bias, which makes it difficult to find any semantic priming effects. Hence, if any semantic priming effects show up in the data, these are robust effects.

Existing words and pseudowords were presented in a pseudorandomised order. We used the same three randomisation lists as in [2], in which the order of the stimuli was manually corrected in case more than six existing words or three pseudowords occurred in succession. Further, [2] avoided rhyme and alliteration between words in consecutive trials.

The materials were recorded in a sound-attenuated booth by [2]. They asked a male speaker of Canadian English to produce the words in clear citation speech, and the words were presented in a randomised order. The members of the target word pairs never occurred in immediate succession to prevent effects of words' semantic relatedness on their realisations. A different native speaker of Canadian English verified that all words were produced in a natural fashion.

\subsection{Procedure}

The procedure was identical to that of Experiment 1 in [2]. The experiment was a self-paced auditory lexical decision task with implicit semantic priming, in which participants had to make a lexical decision, by means of a button press, for each stimu- lus presented. The next trial was presented $1000 \mathrm{~ms}$ after each button press, or after a time-out of $3500 \mathrm{~ms}$. The experiment was conducted in a sound-attenuated booth at the Alberta Phonetics Laboratory. The materials were presented over closed headphones at a comfortable listening level, and the experiment lasted approximately 15 minutes.

\subsection{Results}

Together, the native speakers and bilinguals produced 12495 correct responses, 384 incorrect responses, and 241 time outs. We analysed participants' RTs (from stimulus offset) for the correct responses by means of linear mixed-effects models. As we are primarily interested in the effects of subtle semantic cues, we analysed only the RTs to the target words. All the regression models reported in this study were fitted to the subset of trials for which the response to the preceding trial was also correct. In addition, we excluded outliers for the RTs on the preceding trial to ascertain correct processing of the prime. Thus, our results cannot be explained by listeners' failure to understand the prime or by guessing strategies. Further, we removed data points for which the standardised residuals of the final model were smaller than -2.5 or larger than 2.5 . We took the log of the RTs in order for them to show a normal distribution, and we used a backwards stepwise selection procedure, in which predictors and their interactions were removed if they did not attain significance at the $\alpha<.05$ level. We included the main predictors native language (monolingual or bilingual), lsa (LSA score of the word with its preceding word), target word frequency (log of the word frequency), prime word frequency (log word frequency of the preceding word), and previous $R T$ ( $\log$ of the previous RT, residualised for native language). Further, we included the fixed variables trial number and word $d u$ ration (log of the stimulus duration), and the random variables Participant and Word, in order to reduce the variance in the data. A summary of the results is provided in Table 1.

Table 1: Results for the statistical analysis of the RTs for Experiment 1 .

\begin{tabular}{lccc}
\hline Predictor & $\hat{\beta}$ & $F$ & $p$ \\
\hline \hline Intercept & 12.873 & - & $<.0001$ \\
trial number & -0.002 & 22.09 & $<.01$ \\
target word frequency & -0.212 & 17.64 & $<.01$ \\
lsa & -2.284 & 1.5 & n.s. \\
previous RT & 0.278 & 210.28 & $<.0001$ \\
word duration & -0.789 & 39.81 & $<.0001$ \\
native language & -0.751 & 25.25 & $<.01$ \\
trial number : 1sa & 0.003 & 1.7 & n.s. \\
trial number : native language & 0.002 & 1.13 & n.s. \\
lsa : native language & 0.666 & 0.15 & n.s. \\
target word frequency : lsa & 0.229 & 4.68 & $<.05$ \\
trial number : lsa : native language & -0.003 & 5.9 & $<.05$
\end{tabular}

To begin with, we found an interaction between target word frequency and lsa. This interaction indicates that a stronger semantic relatedness of a target word with its preceding word facilitates the recognition of this target word (e.g., balloon in the word pair airplane - balloon), but only if this target word has an intermediate or low word frequency. For words with a very high word frequency, a stronger semantic relatedness leads to inhibition. This interaction holds for the native and bilingual listeners (for a possible explanation of this interaction, we refer 
to [2]). Hence, the bilinguals show similar patterns to those of the native listeners.

Interestingly, we also found a three-way interaction between native language, lsa and trial number. In order to interpret this interaction, we split the data by native language. We found that bilingual listeners benefited less from semantic cues towards the end of the experiment, whereas there was no such attenuation for the monolingual control group.

In addition, we found shorter RTs if the response for the preceding trial was also fast, for longer words, and for words presented later in the experiment.

These results indicate that Asian-English bilinguals are highly sensitive to subtle semantic cues in English, similar to native listeners, and they use this sensitivity especially in their recognition of low frequency words. However, bilinguals apparently also had more difficulty employing these semantic cues, since they showed smaller semantic priming effects towards the end of the experiment.

This raises the question to what extent bilingual listeners can use semantic cues to process reduced speech, characterised by shorter word durations and missing segments. Previous research has shown that native listeners have difficulty using semantic cues in such adverse listening conditions [2]. We investigated this issue by replicating Experiment 2 of [2], in which participants performed lexical decisions for acoustically reduced words, with bilingual listeners.

\section{Experiment 2}

\subsection{Participants}

Twenty undergraduate students from the University of Alberta, Department of Linguistics participant pool, who had not participated in the previous experiment, received course credit for their participation. The native languages of these bilinguals were Mandarin $(n=7)$, Cantonese $(n=7)$, Korean $(n=5)$, and Japanese $(n=1)$. They were compared to the twenty native speakers of Canadian English tested in Experiment 2 in [2].

\subsection{Materials}

The materials are those of Experiment 2 in [2]. The speaker of Experiment 1 was instructed to produce the word list of Experiment 1 as quickly as possible. Once more, a native speaker of Canadian English verified that all words were produced in a natural fashion, particularly focusing on the way the speaker reduced these words. This recording procedure resulted in reduced words that were shorter than the unreduced materials used in Experiment 1 (673.71 ms versus $388.52 \mathrm{~ms}$ on average, two-tailed t-test: $t(864.8)=46.58, p<.0001)$. In addition, the materials contained some mild segmental reductions: On average, 0.19 segments were deleted per word compared to the realisations of the words in Experiment 1 (range: 0-2 segments per word)

\subsection{Procedure}

The procedure was identical to those of Experiment 1 in the present study and the experiments in [2].

\subsection{Results and discussion}

Together, the native and bilingual participants produced 12317 correct responses, 630 incorrect responses, and 173 time outs. We again analysed the participants' RTs by means of linear mixed-effects models, using the same procedure and including the same predictors as for Experiment 1. A summary of the results is provided in Table 2 .

Table 2: Results for the statistical analysis of the RTs for Experiment 2 .

\begin{tabular}{lccc}
\hline Predictor & $\hat{\beta}$ & $F$ & $p$ \\
\hline \hline Intercept & 10.196 & - & $<.0001$ \\
trial number & -0.0007 & 137.5 & $<.0001$ \\
target word frequency & -0.052 & 11.41 & $<.01$ \\
lsa & 0.085 & 0.127 & n.s. \\
previous RT & 0.326 & 371.44 & $<.0001$ \\
word duration & -0.662 & 31.13 & $<.0001$ \\
language & -0.02 & 7.18 & n.s. \\
lsa : language & -0.328 & 7.24 & $<.05$ \\
trial number : language & 0.0003 & 5.33 & $<.05$
\end{tabular}

The results showed faster responses towards the end of the experiment, for more frequent words, if the previous response was fast, and for longer words. Further, we found a two-way interaction between trial number and native language, which indicates that bilinguals show a larger decrease in reaction times towards the end of the experiment than native listeners.

More importantly, we found an interaction between native language and $l s a$. This interaction indicates that bilingual listeners show smaller semantic facilitation than native listeners, although this facilitation was not statistically significant for native listeners either.

These two experiments show striking differences between the processing of unreduced and reduced pronunciation variants, both by native and Asian-English bilingual listeners. We tested whether these differences attain statistical significance by fitting a regression model to the combined data set of Experiments 1 and 2, using the predictors and their interactions that were present in the final models of Experiments 1 and 2. In addition, we tested for main effects of and interactions with $E x$ periment. A summary of the results is provided in Table 3 .

Table 3: Results for the statistical analysis of the RTs for the combined data set of Experiments 1 and 2.

\begin{tabular}{lccc}
\hline Predictor & $\hat{\beta}$ & $F$ & $p$ \\
\hline \hline Intercept & 7.177 & - & $<.0001$ \\
trial number & -0.0007 & 115.52 & $<.0001$ \\
target word frequency & -0.165 & 17.59 & $<.01$ \\
lsa & -1.238 & 0.81 & n.s. \\
previous RT & 0.294 & 527.95 & $<.0001$ \\
word duration & -0.692 & 148.85 & $<.0001$ \\
Experiment & -0.23 & 18.17 & $<.01$ \\
language & -0.086 & 28.64 & n.s. \\
trial number : language & 0.0003 & 0.006 & n.s. \\
trial number : Experiment & 0.001 & 6.58 & $<.05$ \\
Experiment : language & -0.078 & 3.57 & n.s. \\
lsa : language & -0.21 & 4.58 & $<.05$ \\
target word frequency : lsa & 0.177 & 3.91 & $<.05$ \\
trial number : Experiment : language & -0.0005 & 5.31 & $<.05$
\end{tabular}

First of all, we found a main effect of Experiment, which shows that listeners respond faster to unreduced than to reduced words. Interestingly, we found an interaction between target word frequency and $l s a$, which suggests that this interaction was also present in the data from Experiment 2, although this did not 
appear from the analysis of just the data for Experiment 2. Apparently, it only surfaces after increasing the sample size. More importantly for our research question, we found a two-way interaction between native language and $l s a$, rather than a threeway interaction with Experiment. This finding indicates that, after combining the data sets of Experiments 1 and 2 (thereby increasing the statistical power), bilingual listeners overall show significantly smaller semantic facilitation compared to the native listeners. Finally, we found an interaction between trial number, Experiment, and native language, which indicates that monolingual listeners especially show faster RTs compared to bilingual listeners towards the end of Experiment 1. These results indicate that bilingual listeners are generally less sensitive to subtle semantic cues than native listeners, even though they clearly use them in the processing of unreduced pronunciation variants.

Finally, our results show similar word frequency effects in L1 and L2 speech processing, in line with [14]. Our study extends the finding reported in [14], by showing that these frequency effects are not only similar for unreduced, but also for reduced speech.

\section{General discussion}

The present study investigated Asian-English bilinguals' sensitivity to subtle semantic cues in the processing of unreduced and reduced pronunciation variants. We selected Asian-English bilinguals because these listeners' native and non-native languages are typologically distinct. Moreover, these languages share few cognates, which implies that semantic priming for the bilinguals will show up only if the speakers know the English words (rather than just their native language counterparts). We conducted two auditory lexical decision experiments with implicit semantic priming, in which listeners had to make a lexical decision for every word presented, and consecutive words differed in their semantic relatedness from mildly related to highly related.

First of all, bilingual listeners showed patterns that are very similar to those of native listeners. Bilinguals are sensitive to subtle semantic cues, and they use these cues especially to recognise words with a lower frequency of occurrence. Hence, bilinguals' sensitivity to semantic cues in the non-native language is not restricted to distinctions between semantically related and unrelated words, but it also includes more fine-grained information about the extent to which words in a non-native language are semantically related. Moreover, our results indicate that this sensitivity generalises to bilinguals whose L1 and L2 language are typologically distinct and share few cognates. Further research has to show whether this fine-grained information is due to direct L1-L2 transfer, or whether bilinguals maintain a different semantic network for the non-native language.

Importantly, however, we also found that bilinguals in general show smaller semantic facilitation effects than native listeners. This finding indicates that bilingual listeners have more difficulty than native listeners in using subtle semantic cues in their L2, not only when listening to conversational speech, as may be hypothesised on the basis of [1], but also in their processing of clear speech. Further research is required to test whether bilinguals can benefit from semantic cues in actual face-to-face conversations, in which they can use more sources of information (e.g., the larger discourse, visual information, etc.), or whether listeners' use of such higher level information remains less effective in bilingual speech comprehension.

To conclude, our results show that bilingual listeners are sensitive to subtle semantic cues in their processing of unreduced speech in a typologically distinct non-native language. However, compared to native listeners, they have more difficulty using these cues in the processing of both reduced and clear speech, which possibly affects the extent to which they benefit from such cues in everyday listening situations.

\section{Acknowledgements}

This research was partly funded by a European Young Investigator Award to the third author.

\section{References}

[1] A. R. Bradlow and J. A. Alexander, "Semantic and phonetic enhancements for speech-in-noise recognition by native and nonnative listeners," Journal of the Acoustical Society of America, vol. 121, no. 4, pp. 2339-2349, 2007

[2] M. Van de Ven, B. Tucker, and M. Ernestus, "Semantic context effects in the recognition of acoustically unreduced and reduced words," in Proceedings of the 10th Annual Conference of the International Speech Communication Association, Brighton, 2009, pp. 1867-1870.

[3] K. Johnson, "Massive reduction in conversational American English," in Spontaneous speech: data and analysis. Proceedings of the 1st session of the 10th international symposium. Tokyo, Japan: The National International Institute for Japanese Language, 2004, pp. 29-54.

[4] M. Ernestus, Voice assimilation and segment reduction in casual Dutch, a corpus-based study of the phonology-phonetics interface. Utrecht: LOT, 2000.

[5] B. Schuppler, M. Ernestus, O. Scharenborg, and L. Boves, "An Automatic Method to Analyze Acoustic Reduction in a Corpus of Conversational Dutch," submitted for publication.

[6] M. Ernestus, R. Baayen, and R. Schreuder, "The recognition of reduced word forms," Brain and Language, vol. 81, pp. 162-173, 2002.

[7] M. Van de Ven, M. Ernestus, and R. Schreuder, "Contextual influences in the understanding of spontaneous speech," submitted for publication.

[8] A. Hahne and A. D. Friederici, "Processing a second language: late learners' comprehension mechanisms as revealed by eventrelated brain potentials," Bilingualism: Language and Cognition, vol. 4, pp. 123-141, 2001.

[9] L. H. Mayo, M. Florentine, and S. Buus, "Age of second-language acquisition and perception of speech in noise," Journal of Speech, Language, and Hearing Research, vol. 40, pp. 686-693, 1997.

[10] S. Deerwester, S. T. Dumais, G. W. Furnas, T. K. Landauer, and R. Harshman, "Indexing by latent semantic analysis." Journal of the American Society For Information Science, vol. 41, pp. 391407, 1990.

[11] T. Landauer and S. Dumais, "A solution to Plato's problem: The latent semantic analysis theory of acquisition, induction and representation of knowledge," Psychological Review, vol. 104, no. 2, pp. 211-240, 1997.

[12] T. K. Landauer, P. W. Foltz, and D. Laham, "Introduction to latent semantic analysis," Discourse Processes, vol. 25, pp. 259-284, 1998.

[13] M. Davies, "The Corpus of Contemporary American English (COCA): 385 million words, 1990-present." [On-line], Available: http://www.americancorpus.org., 2008.

[14] S. Imai, A. C. Walley, and J. E. Flege, "Lexical frequency and neighborhood density effects on the recognition of native and spanish-accented words by native english and spanish listeners," Journal of the Acoustical Society of America, vol. 117, no. 2, pp. 896-907, 2005. 